\title{
EPIDEMIOLOGICAL REVIEW OF SURGICAL PROCEDURES PERFORMED IN MALIGNANT HISTOPATHOLOGICAL FINDINGS IN BRASÍLIA, CAPITAL OF BRAZIL
}

Lara D. C. Cavalcante1, Ana P. Fontana1', Arthur L. Seabra', Gabriel C. da Costa', Rafaela A. D. de Oliveira', Yasser N. Abed¹ ${ }^{1}$ Faculdade de Medicina, Universidade de Rio Verde - Rio Verde (GO), Brazil.

Objectives: This study aims to estimate the surgical procedures used to approach hystopal lesions with malignancy in women, according to the age group, in the city of Brasilia during the period from September 2009 to July 2015. Methodology: This study was carried out through research in the country's unified health system (SUS, acronym in Portuguese) database, SISMAMA tabnet. Results: Age groups ranging from 20 to over 70 years were selected in the mentioned period, generating a total of 1,103 surgical procedures performed. Women between the ages of 45-49 represent the age group with the highest number of procedures 17.31\% (191) of the total, with Core Biopsy (CB) being the most common, 29.84\% (57) the total and secondly the incisional biopsy with $9.2 \%$ (18). The second age group with the highest number of procedures is the one that includes women between 50-54 years of age with $15.23 \%$ (168) procedures, being CB also more prevalent with 33.92\% (57), followed by incisional biopsy (IB) and simple mastectomy (SM), 7.14\% (12). In third place, it stands out women between 60-64 years and those with more than 70, each age group responsible for $12.60 \%$ (139) cases, in both CB was the preferred form of approach with a total of $28.05 \%$ (39) and 39.56\% (55) respectively, followed by IB $12.94 \%$ (18) and in women older than 70 , the second procedure was the segmental resection $10.79 \%$ (15). Conclusion: There is a gap in the country's data system, since the most recent data are from 2015. With this we can conclude that the government needs to do a better approach to encourage corroboration with the data. After the epidemiological analysis, the highest number of malignant findings was observed in women aged 45 to 49 years, with the surgical approach being preferred to CB followed by IB. 